Claremont Colleges

Scholarship@ Claremont

All HMC Faculty Publications and Research

HMC Faculty Scholarship

$1-1-1988$

\title{
Ab Initio Investigation of Several Low-lying States of All-trans Octatetraene
}

Robert J. Cave

Harvey Mudd College

Ernest R. Davidson

Indiana University - Bloomington

\section{Recommended Citation}

Cave, R.J.; Davidson, E.R. "An Ab Initio Investigation of Several Low-Lying States of all- trans Octatetraene," J. Phys. Chem. 1988, 92, 2173. doi: 10.1021/j100319a019

This Article is brought to you for free and open access by the HMC Faculty Scholarship at Scholarship @ Claremont. It has been accepted for inclusion in All HMC Faculty Publications and Research by an authorized administrator of Scholarship @ Claremont. For more information, please contact scholarship@cuc.claremont.edu. 


\title{
Ab Initio Investigation of Several Low-Lying States of all-trans-Octatetraene
}

\author{
Robert J. Cave and Ernest R. Davidson* \\ Department of Chemistry, Indiana University, Bloomington, Indiana 47405 (Received: October 26, 1987)
}

\begin{abstract}
The results of $a b$ initio calculations that examine vertical and nonvertical transitions to several low-lying states of alltrans-octatetraene are presented. It is found that the lowest vertical excitation is to a valence $\pi \rightarrow \pi^{*}{ }^{1} \mathrm{~B}_{\mathrm{u}}$ state, and the nominally doubly excited $2^{1} \mathrm{~A}_{\mathrm{g}}$ state occurs approximately $0.4 \mathrm{eV}$ higher at the geometry of the ground state. Using estimated excited-state equilibrium geometries, we find that the $2^{1} \mathrm{~A}_{\mathrm{g}}$ state is indeed the lowest singlet excited state, having a $0-0$ transition energy of $4.15 \mathrm{eV}$. The $0-0$ transition energy for the $1^{1} \mathrm{~B}_{u}$ state is calculated to be $4.56 \mathrm{eV}$. The present results are compared with past theoretical and experimental results.
\end{abstract}

\section{Introduction}

The spectroscopy of octatetraene and its derivatives has been a fruitful ground for both theory and experiment. A derivative of octatetraene was the first compound for which a lowest lying singlet state having the same symmetry as the ground state was observed. ${ }^{1}$ Semiempirical theoretical results gave support to this interpretation of the experiment, characterizing the state as "doubly excited" relative to the ground state. ${ }^{2}$ Since then a host of experiments have been carried out on octatetraene, in the gas phase, solution, and matrices. ${ }^{3-12}$

The lowest optically allowed state of octatetraene occurs at 4.41 $\mathrm{eV}$ in the gas phase, $3,6,7,10$ and in solution it is found to be relatively sensitive to the solvent dielectric constant. ${ }^{3,4}$. This state has been assigned as a ${ }^{1} B_{u} \pi \rightarrow \pi^{*}$ state. In the gas phase under free-jet expansion conditions the lines of the ${ }^{1} B_{u}$ spectrum have been shown to have widths consistent with rather short excited-state lifetimes. ${ }^{6,10}$ This suggests the possibility of geometric distortions leading to radiationless decays to lower lying states In solution and solids, the lowest singlet transition $(0-0)$ has been identified as occurring to a state of the same symmetry as the ground state $\left({ }^{1} A_{g}\right) .3,4,5,11,12$ This conclusion is based on the large gap separating the absorption and emission bands, the asymmetry between the bands, and direct demonstration of the two-photon-allowed, one-photon-forbidden nature of the lowest state. ${ }^{5}$ However, it is interesting to note that gas-phase spectra give a decidedly different picture of the level ordering of octatetraene. The gas-phase fluorescence spectrum shows no Stokes shift relative to the absorption spectrum, ${ }^{3}$ and no evidence for a low-lying ${ }^{1} \mathrm{~A}_{\mathrm{g}}$ state was obtained in electron-energy-loss spectra in the gas phase. Yet, the gas-phase fluorescence lifetime is still much longer than one expects for an allowed transition returning to the ground state. ${ }^{3}$

Other questions regarding the nature of the low-lying states remain open as well. For example, the lowest optically allowed states of butadiene and hexatriene are found theoretically to be somewhat more diffuse than true valence states ${ }^{15,16}$ (more so for butadiene than hexatriene). Whether this diffuse character persists for octatetraene is an open question. A second question is that of the ordering of the low-lying states in a vertical transition from the ground state. For butadiene and hexatriene recent $a b$ initio results ${ }^{15,16}$ have shown that the lowest vertical transition occurs to the $1^{1} B_{u}$ state and that the $2^{1} A_{g}$ state is from 0.4 to $0.6 \mathrm{eV}$ higher at the ground-state geometry. For octatetraene the level ordering at the ground-state geometry is not known, although the $1^{1} B_{u}$ and $2^{1} A_{g} 0-0$ lines are separated by approximately $0.75 \mathrm{eV}^{3,4}$

In this article we report results from ab initio calculations on all-trans-octatetraene (hereafter octatetraene). We believe these are the first $a b$ initio results presented for excited states in this system. The methods are similar to those employed for our studies of butadiene and hexatriene. Our focus will be on determining the nature of the two lowest lying singlet states and their ordering in both vertical and nonvertical transitions from the ground state. We also present results for the lowest triplet state, a low-lying Rydberg state, and the lowest cation of octatetraene.

\footnotetext{
* Author to whom correspondence should be addressed.
}

\section{Theoretical Methods}

The molecule is taken to have $C_{2 h}$ symmetry and is chosen to lie in the $y z$ plane; thus the $x$ axis is the $C_{2}$ axis. The estimated equilibrium geometry was taken from Lasaga et al., ${ }^{17}$ which was optimized for the ground state of octatetraene. Where parameters were not explicitly stated for octatetraene we have used the analogous ground-state values obtained for butadiene in ref 17 , except that the $\mathrm{C}-\mathrm{H}$ bond lengths were taken as $1.10 \AA$. The results of ref 17 were approximately $0.01-0.02 \AA$ shorter than the experimental estimates of the $\mathrm{C}-\mathrm{H}$ bond lengths for butadiene and hexatriene. We have chosen the above value to account for this. For the calculations concerning nonvertical excitation energies the estimated geometries were obtained as follows. The $\mathrm{C}-\mathrm{C}$ and $\mathrm{C}=\mathrm{C}$ bond lengths were taken as the lengths optimized for the $1^{1} B_{u}$ and $2^{1} A_{g}$ states from ref 9 and 17 , respectively. All bond angles and $\mathrm{C}-\mathrm{H}$ bond lengths were held fixed at the values used for the $1^{1} \mathrm{~A}_{\mathrm{g}}$ (ground) state. The $\mathrm{C}-\mathrm{H}$ bond lengths and all angles were not significantly different between the ground state and the excited states, and it was felt for conceptual simplicity we would focus on the effects of the changes in the carbon skeleton.

The basis sets used are as follows. For carbon we have taken the Dunning/Hay ${ }^{18}(3 \mathrm{~s}, 2 \mathrm{p})$ contraction of the Huzinaga ${ }^{19}(9 \mathrm{~s}, 5 \mathrm{p})$

(1) Hudson, B. S.; Kohler, B. E. Chem. Phys. Lett. 1972, 14, 299.

(2) Schulten, K.; Karplus, M. Chem. Phys. Lett. 1972, 14, 305.

(3) Gavin, R. M., Jr.; Weisman, C.; McVey, J. K.; Rice, S. A. J. Chem. Phys. 1978, 68, 522 .

(4) D'Amico, K. L.; Manos, C.; Christensen, R. L. J. Am. Chem. Soc. 1980, 102, 1777 .

(5) Granville, M. F.; Holtom, G. R.; Kohler, B. E. J. Chem. Phys. 1980 , 72,4671 .

(6) Heimbrook, L. A.; Kenny, J. E.; Kohler, B. E.; Scott, G. W. J. Chem. Phys. 1981, 75, 4338 .

(7) Allan, M.; Neuhaus, L.; Haselbach, E. Helv. Chim. Acta 1984, 67, 1776 .

(8) Lurito, J. T.; Dunkin, I. R.; Andrews, L. J. Mol. Spectrosc. 1985, 114, 54.

(9) Kohler, B. E.; Spiglanin, T. A.; Hemley, R. J.; Karplus, M. J. Chem. Phys. 1984, 80, 23. 4210 .

(10) Leopold, D. G.; Vaida, V.; Granville, M. F. J. Chem. Phys. 1984, 81,

(11) Fang, H. L. B.; Thrash, R. J.; Leroi, G. E. J. Chem. Phys. 1977, 67, 3389.

(12) Itoh, T.; Kohler, B. E. J. Phys. Chem. 1987, 91, 1760.

(13) Nascimento, M. A. C.; Goddard, W. A., III Chem. Phys. 1979, 36, 147.

(14) (a) Nascimento, M. A. C.; Goddard, W. A., III Chem. Phys. Lett. 1979, 60, 197. (b) Nascimento, M. A. C.; Goddard, W. A., III Chem. Phys. $1980,53,265$.

(15) Cave, R. J.; Davidson, E. R. J. Phys. Chem. 1987, 91, 4481

(16) Cave, R. J.; Davidson, E. R. J. Phys. Chem. 1988, 92, 614. 5230 .

(17) Lasaga, A. C.; Aerni, R. J.; Karplus, M. J. Chem. Phys. 1980, 73,

(18) Dunning, T. H., Jr.; Hay, P. J. In Methods of Electronic Structure Theory; Schaefer, H. F., III, Ed.; Plenum: New York, 1977; Vol. III, p 1.

(19) Huzinaga, S. J. Chem. Phys. 1965, 42, 1293. 
basis. This was augmented with a single $d$ function on each carbon (exponent $=0.55$ ) and a single $\mathrm{p}$ Rydberg function on each carbon (exponent $=0.021)$. For $H$ the Dunning/Huzinaga ${ }^{20}(4 s / 2 s)$ set was used. In a few calculations we used the above basis augmented with diffuse $p$ Rydberg functions (exponent $=0.09$ ) placed on carbons three and five. Unless otherwise specified, however, the nonaugmented basis was used. In all cases the in-plane components of the $d$ and $p$ Rydberg functions were discarded. Using this basis in $\mathrm{CI} 2$ calculations (including differential $\sigma \sigma^{\prime}$ excitations; see ref 15,16 , and 21) on the excitation energy for the lowest $\pi$ $\rightarrow \pi^{*}$ state of ethylene, we obtained a value of $8.19 \mathrm{eV}$. For comparison, using $\mathrm{TZ}$ bases for $\mathrm{H}$ and $\mathrm{C}^{15}$ augmented with two sets of $p$ Rydberg functions (exponents $=0.021,0.009$ ), a single $s$ Rydberg function (exponent $=0.009$ ), and a set of $d$ polarization functions (exponent $=0.55$ ), we obtained an excitation energy of $8.16 \mathrm{eV}$. In the latter calculation the in-plane components of the $d$ and $p$ Rydberg functions were not discarded. Finally, one can compare this result with previous results ${ }^{21}$ which used a DZ basis for $\mathrm{C}$ and $\mathrm{H}$, augmented with two sets of diffuse $\mathrm{d}$ Rydberg functions, and polarization functions on $\mathrm{C}$ and $\mathrm{H}$ and obtained $7.96 \mathrm{eV}$ for the $\mathrm{CI} 2$ excitation energy. Thus, the above basis should be adequate for the present purposes. Below we gauge its accuracy for octatetraene by comparison with experimental results. For convenience we designate basis functions (or MO's) which are symmetric with respect to reflection in the molecular plane as $\sigma$; those which are antisymmetric we designate as $\pi$.

All SCF, CI, and molecular property results were obtained by using the MELD suite of electronic structure codes developed in the laboratory. ${ }^{22}$ Properties are calculated as the expectation value of the final (selected, see below) CI wave function. Where natural orbitals were not used (all states except the ${ }^{1} B_{u}$ states) the virtual space was transformed to the K orbitals (KO) of Feller and Davidson. ${ }^{23}$ We performed two general types of CI's for the states examined below. The first, designated CI8 (analogous to the $\mathrm{CI} 2,{ }^{21} \mathrm{CI} 4,{ }^{15}$ and $\mathrm{CI} 6^{16}$ treatments used previously), allows all single excitations from the chosen reference configurations along with all double excitations involving at least one of the $\pi$ electrons. No excitations were allowed from the four lowest $a_{g}$ and $b_{u}$ orbitals; these corresponded to $\mathrm{C} 1 \mathrm{~s}$ orbitals. No $\sigma \sigma^{\prime}$ double excitations were allowed..$^{15,16}$ We also performed CI4 calculations for a number of the states of interest in which only double excitations involving at least one of the four highest lying $\pi$ electrons were allowed. The second general type of CI used was one in which up to quadruple excitations in the $\pi$ space were allowed relative to the SCF configuration of interest. For calculations on the $2^{1} \mathrm{~A}_{\mathrm{g}}$ state up to quadruple excitations were allowed from the ground-state SCF configuration. No $\sigma$ excitations were allowed. We designate these results as $\pi \mathrm{QCI}$ below. In a previous study the $\pi \mathrm{QCI}$ treatment was shown to yield good agreement with calculations allowing $\sigma \pi$ correlation for the $2^{1} A_{g}$ and $1^{3} B_{u}$ states of hexatriene. ${ }^{16}$

In all cases perturbation theory was used to select configurations to be treated in the CI's. ${ }^{15,16,24}$ In the results below we report the percentage of the second-order perturbation-theory energy accounted for by the given subset of configurations retained variationally (PTK = percentage of the perturbation-theory energy kept). In the $\pi \mathrm{QCI}$ calculations, relatively large zeroth-order spaces were used for perturbation theory selection to ensure the inclusion of the dominant triple and quadruple excitations. Only in the CI8 calculations presented below were all single excitations relative to the reference functions kept in the variational portion of the calculation. In addition, in the CI8 calculations we have corrected the final energies for size-inconsistency effects using a perturbation-theory-based estimate of the unlinked cluster ef-

(20) Dunning. T. H., Jr. J. Chem. Phys. 1970, 53, 2823.

(21) McMurchie, L. E.; Davidson, E. R. J. Chem. Phys. 1977, 66, 2959.

(22) The MELD series of electronic structure codes was developed by $L$. E. McMurchie, S. T. Elbert, S. R. Langhoff, and E. R. Davidson and was extensively modified by D. Feller and D. C. Rawlings.

(23) Feller, D.; Davidson, E. R. J. Chem. Phys. 1981, 74, 3977.

(24) Rawlings, D. C.; Davison, E. R.; Gouterman, M. Int. J. Quantum Chem. 1984, 26, 251 .
TABLE I: SCF Results for Various States of Octatetraene

\begin{tabular}{cccc}
\hline & state & energy, hartree & $\Delta E, \mathrm{eV}$ \\
\hline neutral & $1^{1} \mathrm{~A}_{\mathrm{g}}$ & -308.6341 & \\
& $1^{3} \mathrm{~B}_{\mathrm{u}}$ & -308.5437 & 2.46 \\
& $1^{1} \mathrm{~B}_{\mathrm{u}}$ & -308.4404 & 5.27 \\
cation & $1^{2} \mathrm{~B}_{\mathrm{g}}$ & -308.3845 & 6.79
\end{tabular}

fects. ${ }^{24}$ These results are designated below as $+\mathrm{SC}$. For the $\pi \mathrm{QCI}$ results the zeroth-order space used for perturbation-theory selection was large enough so that most of the important unlinked terms should have been incorporated into the variational treatment. We have thus performed no size-consistency correction for the $\pi \mathrm{QCI}$ results. The extrapolation and size-consistency correction are expected to be accurate to within $10 \%$. For the results presented here this amounts to uncertainties of $0.2-0.4 \mathrm{eV}$ in the energies reported. This is in addition to any errors incurred by the use of truncated CI's or the incompleteness of our basis sets.

As in previous studies, ${ }^{15,16}$ we have used average natural orbitals ${ }^{25}$ (ANO) obtained from a small preliminary CI (CI1 ${ }^{15,16,21}$ ) on the two lowest ${ }^{1} \mathrm{~B}_{\mathrm{u}}$ states to generate suitable zeroth-order descriptions for these two states. For the smaller polyenes it was found that the SCF description of the $1^{1} B_{u}$ state heavily biased the $C I$ result toward Rydberg character, while the ANO description treated the valence-like and Rydberg-like states on a more nearly equal basis.

\section{Results}

In Table I results from restricted closed- and open-shell Hartree-Fock calculations are presented. The ground-state occupation in the $\sigma$ space is $1-10 \mathrm{a}_{\mathrm{g}}{ }^{2}, 1-9 \mathrm{~b}_{\mathrm{u}}{ }^{2}$, and in the $\pi$ space the occupation is $1 \mathrm{a}_{\mathrm{u}}{ }^{2} 2 \mathrm{a}_{\mathrm{u}}{ }^{2} 1 \mathrm{~b}_{\mathrm{g}}{ }^{2} 2 \mathrm{~b}_{\mathrm{g}}{ }^{2}$. For the remaining states of Table I the $\sigma$ space occupation is the same as that of the ground state while in the $\pi$ space one has ${ }^{3,1} \mathrm{~B}_{\mathrm{u}}: 1 \mathrm{a}_{\mathrm{u}}{ }^{2} 2 \mathrm{a}_{\mathrm{u}}{ }^{2} 3 \mathrm{a}_{\mathrm{u}}{ }^{1} 1 \mathrm{~b}_{\mathrm{g}}{ }^{2} 2 \mathrm{~b}_{\mathrm{g}}{ }^{1}$ and ${ }^{2} \mathrm{~B}_{\mathrm{g}}: 1 \mathrm{a}_{\mathrm{u}}{ }^{2} 2 \mathrm{a}_{\mathrm{u}}{ }^{2} 1 \mathrm{~b}_{\mathrm{g}}{ }^{2} 2 \mathrm{~b}_{\mathrm{g}}{ }^{1}$. No SCF results are presented for the $2^{1} \AA_{\mathrm{g}}$ state since previous studies on the shorter polyenes suggest this will be a multiconfigurational state at zeroth order.

In Table II results are presented from several CI8 calculations. A number of points should be noted. First, the two $1^{1} \mathrm{~A}_{\mathrm{g}}$ results differ only in the number of configurations treated variationally (i.e., the percentage of the total second-order perturbation-theory energy accounted for by the chosen subset of configurations). It is seen that the two energy estimates are quite close. Second, the results for the two ${ }^{1} B_{u}$ states are from a single calculation in which Cl8 configurations were generated for the two lowest ${ }^{1} \mathbf{B}_{u}$ configurations (i.e., ground-state $\sigma$ occupation; $1 \mathrm{a}_{\mathrm{u}}{ }^{2} 2 \mathrm{a}_{\mathrm{u}}{ }^{2} 3 \mathrm{a}_{\mathrm{u}}{ }^{1} 1 \mathrm{~b}_{\mathrm{g}}{ }^{2}$ $2 \mathrm{~b}_{\mathrm{g}}{ }^{1}$ and $1 \mathrm{a}_{\mathrm{u}}{ }^{2} 2 \mathrm{a}_{\mathrm{u}}{ }^{2} 4 \mathrm{a}_{\mathrm{u}}{ }^{1} 1 \mathrm{~b}_{\mathrm{g}}{ }^{2} 2 \mathrm{~b}_{\mathrm{g}}{ }^{1}$ ). An iterative procedure was used to define the zeroth-order space for the ${ }^{1} \mathrm{~B}_{\mathrm{u}}$ states. ${ }^{15,16}$ It is seen that the percentage of the second-order energy accounted for by the configurations retained for the variational part of the calculation is virtually the same for both states. This indicates that the states are being treated equivalently in the variational portion of the calculation. The $\left\langle x^{2}\right\rangle$ values clearly show that the lowest ${ }^{1} B_{u}$ state is a valence state and the $2^{1} B_{u}$ state is a Rydberg state. Similar results were obtained from the CI1 calculations to generate the 'B $B_{u} A N O$ 's. In the CIl calculations an energy separation of $1.61 \mathrm{eV}$ was found between the $1^{1} \mathrm{~B}_{\mathrm{u}}$ and $2^{1} \mathrm{~B}_{\mathrm{u}}$ states and $\left\langle x^{2}\right\rangle$ values of 44.6 and $76.4 \mathrm{au}^{2}$, respectively, were obtained.

Results are also shown in Table II from calculations using the augmented basis described above. This basis has an additional Rydberg function of more diffuse character at carbons three and five. It is seen that the total energies of the $1^{1} \mathrm{~A}_{\mathrm{g}}$ and the $1^{1} \mathrm{~B}_{\mathrm{u}}$ states are essentially unaffected by inclusion of these functions, but the $2^{1} B_{u}$ excitation energy has dropped by almost $0.15 \mathrm{eV}$.

In Table III results from CI4 calculations are presented for the same states considered in Table II. Our aim in these calculations was to examine whether one needed to correlate the more tightly bound $\pi$ electrons to obtain reasonable accuracy. It is seen that the excitation energies so obtained are uniformly higher than the CI8 values, especially for the Rydberg-like $2^{1} B_{u}$ and for the

(25) Bender, C. F.; Schaefer, H. F., III J. Chem. Phys. 1971, 55, 4798 
TABLE II: CI8 Results for Various States of Octatetraene

\begin{tabular}{|c|c|c|c|c|c|c|c|}
\hline state & MO's & $\operatorname{refs}^{a}$ & PTK $^{b}$ & energy, hartree & energy $+\mathrm{SC},{ }^{c}$ hartree & $\Delta E,^{c, d} \mathrm{eV}$ & $\left\langle x^{2}\right\rangle^{c}$ \\
\hline $\begin{array}{l}1^{1} A_{g} \\
1^{1} A_{g} \\
1^{3} B_{u} \\
1^{1} B_{u} \\
2^{1} B_{u} \\
1^{2} B_{g}\end{array}$ & $\begin{array}{l}1^{1} A_{g} \text { KO } \\
1^{1} A_{g} \text { KO } \\
1^{3} B_{u} \text { KO } \\
1^{1} B_{u} \text { ANO } \\
{ }^{1} B_{u} \text { ANO } \\
1^{2} B_{g} \text { KO }\end{array}$ & $\begin{array}{l}1 \\
1 \\
1 \\
2 \\
2 \\
1\end{array}$ & $\begin{array}{l}92.1 \\
84.1 \\
87.8 \\
85.7 \\
85.7 \\
95.1\end{array}$ & $\begin{array}{l}-308.9801 \\
-308.9809 \\
-308.8856 \\
-308.7971 \\
-308.7631 \\
-308.7101\end{array}$ & $\begin{array}{l}-309.0470 \\
-309.0446 \\
-308.9543 \\
-308.8708 \\
-308.8342 \\
-308.7725\end{array}$ & $\begin{array}{l}0.07 \\
2.52 \\
4.79 \\
5.79 \\
7.47\end{array}$ & $\begin{array}{l}42.4 \\
42.3 \\
42.4 \\
44.2 \\
77.1 \\
38.9\end{array}$ \\
\hline $\begin{array}{l}1^{1} \mathbf{A}_{g} \\
1^{1} B_{u} \\
2^{1} B_{u}\end{array}$ & $\begin{array}{l}1^{1} A_{\mathrm{g}} \text { KO } \\
{ }^{1} \mathrm{~B}_{\mathrm{u}} \text { ANO } \\
{ }^{1} \mathrm{~B}_{\mathrm{u}} \text { ANO }\end{array}$ & $\begin{array}{l}1 \\
2 \\
2\end{array}$ & $\begin{array}{l}93.5 \\
85.8 \\
84.9\end{array}$ & $\begin{array}{l}\text { ented Basis } \\
\qquad-308.9803 \\
-308.7955 \\
-308.7742\end{array}$ & $\begin{array}{l}-308.0479 \\
-308.8728 \\
-308.8404\end{array}$ & $\begin{array}{l}4.76 \\
5.65\end{array}$ & $\begin{array}{r}42.4 \\
44.5 \\
107.1\end{array}$ \\
\hline
\end{tabular}

${ }^{a}$ Number of reference configurations. ${ }^{b}$ Percentage of the second-order perturbation-theory energy accounted for by the configurations treated variationally. ${ }^{c}$ Energies corrected by using the size-consistency correction discussed in section II. ${ }^{d}$ Relative to the appropriate $1^{1} \mathrm{~A}_{\underline{g}}$ result. The size-consistency corrected results are used. 'In au².

TABLE III: CI4 Results for Various States of Octatetraene

\begin{tabular}{|c|c|c|c|c|c|c|c|}
\hline state & MO's & $\operatorname{refs}^{a}$ & PTK $^{b}$ & energy, hartree & energy $+\mathrm{SC}_{,}{ }^{c}$ hartree & $\Delta E E_{,}^{c, d} \mathrm{eV}$ & $\left\langle x^{2}\right\rangle^{e}$ \\
\hline $\begin{array}{l}1^{1} A_{g} \\
1^{3} B_{u} \\
1^{1} B_{u} \\
2^{1} B_{u} \\
1^{2} B_{g}\end{array}$ & $\begin{array}{l}1^{1} A_{g} \mathrm{KO} \\
1^{3} B_{u} \mathrm{KO} \\
{ }^{1} B_{u} A N O \\
{ }^{1} B_{u} A N O \\
1^{2} B_{g} \mathrm{KO}\end{array}$ & $\begin{array}{l}1 \\
1 \\
2 \\
2 \\
1\end{array}$ & $\begin{array}{l}98.0 \\
91.6 \\
90.8 \\
90.4 \\
98.3\end{array}$ & $\begin{array}{l}-308.8520 \\
-308.7543 \\
-308.6671 \\
-308.6207 \\
-308.5661\end{array}$ & $\begin{array}{l}-309.8836 \\
-308.7849 \\
-308.7042 \\
-308.6539 \\
-308.5913\end{array}$ & $\begin{array}{l}2.69 \\
4.88 \\
6.25 \\
7.95\end{array}$ & $\begin{array}{l}43.0 \\
42.7 \\
44.1 \\
77.2 \\
39.1\end{array}$ \\
\hline
\end{tabular}

${ }^{a}$ Number of reference configurations. ${ }^{b}$ Percentage of the second-order perturbation-theory energy accounted for by the configurations treated variationally. ${ }^{c}$ Energies corrected by using the size-consistency correction discussed in section II. ${ }^{d}$ Relative to the appropriate $1^{1} \mathrm{~A}_{\mathrm{g}}$ result. The size-consistency corrected results are used. ${ }^{e}$ In $\mathrm{au}^{2}$.

TABLE IV: $\pi$ QCI Results for Octatetraene

\begin{tabular}{ccccc}
\hline state & MO's & $\begin{array}{c}\text { energy, } \\
\text { hartree }\end{array}$ & $\Delta E, \mathrm{eV}$ & $\left\langle x^{2}\right\rangle^{a}$ \\
\hline $1^{1} \mathrm{~A}_{\mathrm{g}}$ & $1^{1} \mathrm{~A}_{\mathrm{g}} \mathrm{KO}$ & -308.7629 & & 42.2 \\
$2^{1} \mathrm{~A}_{\mathrm{g}}$ & $1^{1} \mathrm{~A}_{\mathrm{g}} \mathrm{KO}$ & -308.5716 & 5.21 & 42.3 \\
$1^{3} \mathrm{~B}_{\mathrm{u}}$ & $1^{3} \mathrm{~B}_{\mathrm{u}} \mathrm{KO}$ & -308.6728 & 2.45 & 42.3
\end{tabular}

${ }^{a}$ In $\mathrm{au}^{2}$.

${ }^{2} \mathrm{~B}_{\mathrm{g}}$ cation. One might suggest this difference arises due to the neglect of single excitations relative to the reference function in the CI4 calculations. (Second-order perturbation-theory selection will discard single excitations relative to an RHF reference.) However, in $\mathrm{CI} 8$ calculations where single excitations were only kept if they yielded nonzero energy lowerings at second order, the two ${ }^{1} \mathrm{~B}_{u}$ excitation energies were $0.2 \mathrm{eV}$ lower than those in Table II. The ${ }^{3} B_{u}$ excitation energy rose by only $0.05 \mathrm{eV}$. Thus, the results in Table III strongly suggest that one needs to consider the correlation of all $\pi$ electrons and not merely the few highest lying electrons.

In Table IV results are presented from $\pi \mathrm{QCI}$ calculations for a number of states. In the case of the $2^{1} \mathrm{~A}_{\mathrm{g}}$ state the results are based on using the ground-state MO's. In results for the $2^{1} \mathrm{~A}$ state of butadiene it was found ${ }^{26}$ that a similar calculation yielded essentially exact agreement with either the CI4 results (which allowed $\sigma$-orbital relaxation and $\sigma \pi$ correlation effects) or the MCSCF results (which allowed correlation only in the $\pi$ space but allowed $\sigma$-orbital relaxation) of ref 15 . Similar results were also obtained for the $2^{1} \mathrm{~A}_{\mathrm{g}}$ state of hexatriene. It is seen that the calculated excitation energy to the ${ }^{3} B_{u}$ state is within $0.1 \mathrm{eV}$ of the CI8 result in Table II.

In Table $\mathrm{V}$ results are presented from calculations using the estimated excited-state equilibrium geometries discussed in section II. In CII calculations on the two lowest states of ${ }^{1} \mathrm{~B}_{\mathrm{u}}$ symmetry at the estimated ${ }^{1} \mathbf{B}_{u}$ equilibrium geometry we found the dominantly occupied natural orbitals from the $1^{1} \mathrm{~B}_{u} \mathrm{CI}$ result to be quite similar to the ${ }^{1} B_{4}$ SCF occupied orbitals. This being the case, we chose to use the $1^{1} B_{u} S C F / K O$ orbitals directly, rather than using the CII ANO's.

It is seen that the calculated $0-0$ transition energy for the $1{ }^{1} B_{u}$ state is $0.23 \mathrm{eV}$ lower than the vertical excitation energy from Table II. The $2^{1} \mathrm{~A}_{\mathrm{g}}$ state drops by a larger amount in going to

(26) Cave, R. J.; Davidson, E. R., unpublished results. its estimated equilibrium geometry; so much so that the estimated $0-0$ transition energy is significantly below that of the $1^{1} B_{u}$ state. If one assumes that the fluorescence intensity maximum corresponds to the vertical transition from the $2^{1} \mathrm{~A}_{\mathrm{g}}$ minimum, one obtains $3.74 \mathrm{eV}$ for the position of the fluorescence intensity maximum.

\section{Discussion}

A summary of experimentally determined excitation energies is presented in Table $\mathrm{V}$, along with previous theoretical estimates. Examining first the vertical excitation energies to the ${ }^{3} \mathrm{~B}_{\mathrm{u}}$ state, it is seen that our $\pi \mathrm{QCI}$ result is high by $0.35 \mathrm{eV}$ and our $\mathrm{CI} 8$ result is high by $0.41 \mathrm{eV}$. (We do not consider the CI4 results since we believe they are less accurate than the CI8 results, for reasons mentioned in section III.) Several factors may contribute to this error. One is basis set inadequacy. We noted in section II that the present basis yields energies for the $\pi \rightarrow \pi^{*}$ state of ethylene that are somewhat higher than a basis which includes polarization functions on the hydrogens. Errors on the order of $0.2 \mathrm{eV}$ were found for the lowest ${ }^{3} \mathrm{~B}_{\mathrm{u}}$ state of hexatriene ${ }^{16}$ with a basis which also did not include $\mathrm{H}$ atom polarization functions. Another possibility is that the present geometry for the ground state is not as accurate as those used for the smaller polyenes. This would demand that the excited states examined here be more sensitive to geometric variations than the ground state. Evidence exists for this for both the ${ }^{3} \mathrm{~B}_{u}{ }^{27}$ and the $2^{1} \mathrm{~A}_{\mathrm{g}}$ state. ${ }^{17,27}$ It is interesting to note that the electron-energy-loss spectrum ${ }^{7}$ shows a $0.4-\mathrm{eV}$ difference between the $0-0$ transition energy and the intensity maximum (assumed vertical excitation) for the ${ }^{3} B_{u}$ state, whereas the $0-0$ transition and the intensity maximum coincide for the ${ }^{1} B_{u}$ state.

For the valencelike $1^{1} B_{u}$ state we obtain a vertical excitation energy of $4.79 \mathrm{eV}(4.76 \mathrm{eV}$ in the augmented basis) and a $0-0$ transition energy based on the approximate excited-state equilibrium geometry of $4.56 \mathrm{eV}$. (Correcting the $0-0$ energy for the difference between the augmented and nonaugmented basis sets gives a $0-0$ energy of $4.53 \mathrm{eV}$.) This contrasts with the interpretation of previous experimental results for this state which uniformly find the intensity maximum to occur at the 0-0 frequency, $4.41 \mathrm{eV}$, and as a result assign this peak to the vertical transition. However, we believe this assignment may be incorrect. 580 .

(27) Said, M.; Maynau, D.; Malrieu, J.-P. J. Am. Chem. Soc. 1984, 106, 
TABLE V: Results for Estimated Excited-State Geometries

\begin{tabular}{|c|c|c|c|c|c|c|c|c|}
\hline state & geometry $^{a}$ & MO's & ref & PTK $^{b}$ & energy, hartree & $\begin{array}{l}\text { energy }+ \\
\mathrm{SC}^{c} \text { hartree }\end{array}$ & $\Delta E\left(1^{1} \mathrm{~A}_{\mathrm{g}}, \text { eq }\right)^{d}$ & $\left\langle x^{2}\right\rangle^{a}$ \\
\hline \multicolumn{9}{|c|}{ CI8 Calculations } \\
\hline $1^{1} B_{u}$ & $1^{1} B_{u}$ & $1^{1} \mathrm{~B}_{\mathrm{u}} \mathrm{KO}$ & 1 & 92.1 & -308.8076 & -308.8794 & 4.56 & 44.7 \\
\hline \multicolumn{9}{|c|}{$\pi \mathrm{QCI}$ Calculations } \\
\hline $1^{1} \mathrm{~A}_{8}$ & $2^{1} \mathrm{Ag}_{\mathrm{g}}$ & $1^{1} \mathrm{~A}_{\mathrm{g}} \mathrm{KO}$ & & 98.0 & -308.7476 & & 0.42 & 42.6 \\
\hline $2^{1} \mathrm{~A}_{\mathrm{g}}$ & $2^{1} A_{g}^{g}$ & $1^{1} \mathrm{~A}_{\mathrm{g}}^{\mathrm{B}} \mathrm{KO}$ & & 97.1 & -308.6103 & & 4.15 & 42.4 \\
\hline
\end{tabular}

${ }^{a}$ Estimated excited-state geometry used in the calculation. ${ }^{b}$ Percentage of the second-order perturbation-theory energy accounted for by the configurations treated variationally, ${ }^{c}$ Energies corrected by using the size-consistency correction discussed in section II. ${ }^{d}$ In $\mathrm{eV}$; relative to the appropriate $1^{1} \mathbf{A}_{\mathbf{g}}$ result at the ground-state equilibrium geometry in the nonaugmented basis. For the ${ }^{1} \mathrm{~B}_{\mathrm{u}}$ result the $+\mathrm{SC}$ values are used. ${ }^{e}$ In au ${ }^{2}$.

The present $a b$ initio results and past semiempirical results do find a significant decrease in energy for the $1^{1} \mathrm{~B}_{\mathrm{u}}$ state in proceeding from the ground-state geometry to that of the ${ }^{1} \mathrm{~B}_{\mathrm{u}}$ state. This suggests that there should be a difference between the $0-0$ and vertical transition energies. Indeed, while the experimental results obtain the intensity maximum at $4.41 \mathrm{eV}$, there are peaks of significant intensity at $4.61(60 \% 0-0)$ and $4.82(16 \% 0-0) \mathrm{eV} .^{10}$ If the $1^{1} \mathrm{~B}_{\mathrm{u}}$ state at the ground-state geometry is somewhat more Rydberg-like than at the equilibrium geometry for the excited state, the largest intensity peaks in the spectrum could occur for nonvertical geometries. While the present results do not indicate a substantial difference in spatial extent for the $1^{1} B_{u}$ state between the two geometries, this may be due to the tendency of the CI8 calculations to underestimate the spatial extent of the ${ }^{1} B_{u}$ state when compared with results where the $\sigma$ electrons are correlated more extensively. ${ }^{15}$ In any event, the current results do find the lowest $1^{1} \mathbf{B}_{u}$ state to occur in the region observed experimentally and to be essentially valencelike, in agreement with the fact that the transition is observed in liquids and solids..$^{3-5}$

The present calculations for the $2^{1} \mathrm{~A}_{\mathrm{g}}$ state obtain a vertical excitation energy of $5.21 \mathrm{eV}$, a $0-0$ transition energy of $4.15 \mathrm{eV}$, and an estimated fluorescence intensity maximum (based on a vertical transition to the ground state) of $3.74 \mathrm{eV}$. The calculated 0-0 transition energy is $0.56 \mathrm{eV}$ higher than the value obtained from extrapolation of the solution results to the gas phase. ${ }^{3,4}$ The error may be the result of an inaccurate guess for the excited-state geometry, inadequate treatment of the correlation problem, or use of the ground-state MO's to describe the excited state at its equilibrium geometry. Taking the error in the 0-0 transition energy $(0.56 \mathrm{eV})$ as a correction for the remaining $2^{1} \mathrm{~A}_{\mathrm{g}}$ excitation energies leads to an estimate of the fluorescence intensity maximum of $3.18 \mathrm{eV}$, in excellent agreement with the experimental value of $3.19 \mathrm{eV},{ }^{3}$ Correcting the vertical excitation energy by the same amount leads to an estimated $E_{\text {vert }}$ of $4.65 \mathrm{eV}$, significantly higher than the inferred value of $3.97 \mathrm{eV} .{ }^{29}$ However, if the principal error in the calculated $0-0$ transition energy arises from an inaccurate excited-state geometry, the correction should not be used for the $2^{1} \mathrm{~A}_{\mathrm{g}}$ vertical excitation energy, and the calculated value of $5.21 \mathrm{eV}$ would be more appropriate. Thus, we view this estimate $\left(E_{\text {vert }}=4.65 \mathrm{eV}\right)$ as a lower bound for the $2^{1} \mathrm{~A}_{\mathrm{g}}$ vertical excitation energy.

We find that at the ground-state geometry the $2^{1} \mathrm{~A}_{\mathrm{g}}$ state is either $0.44 \mathrm{eV}$ above the $1^{1} \mathrm{~B}_{\mathrm{u}}$ state (assuming the $2^{1} \mathrm{~A}_{\mathrm{g}} 0-0$ correction is not appropriate for the vertical transition) or $0.1 \mathrm{eV}$ below the $1^{1} B_{u}$ state. We are in disagreement with semiempirical studies which found the $2^{\mathrm{I}} \mathrm{A}_{\mathrm{g}}$ state significantly below the $1{ }^{1} \mathbf{B}_{4}$ state at the ground-state geometry..$^{17,28}$ We have argued previously ${ }^{16}$ that this discrepancy arises from the parameters chosen in such semiempirical schemes, which are optimized to treat the ionic valence-bond states (i.e., the ${ }^{1} B_{u}$ states). In previous $a b$ initio studies $^{15,16}$ it has been shown that the correct description of the ionic states requires extensive treatment of $\sigma \pi$ correlation. In a semiempirical $\pi$-electron-only model this means that the choice of $\pi$-electron parameters must compensate for the neglect of $\sigma$-electron effects. However, for the covalent states, such as the

(28) Tavan, P.; Schulten, K. J. Chem. Phys. 1979, 70, 5407.

(29) Hudson, B. S.; Kohler, B. E.; Schulten, K. In Excited States; Lim, E. C., Ed.; Academic: New York, 1982; Vol. VI, p 1.
TABLE VI: Comparison with Previous Results

\begin{tabular}{llll}
\hline state & & \multicolumn{1}{c}{$\Delta E, \mathrm{eV}$} & \multicolumn{1}{c}{ ref } \\
\hline $1^{3} \mathrm{~B}_{\mathrm{u}}$ & experiment & $1.7(0-0), 2.1(\mathrm{v})$ & 7 \\
& eff Hamiltonian & $1.57(0-0), 2.07(\mathrm{v})$ & 27 \\
& ab initio & $2.5(\mathrm{v})$ & $\mathrm{b}$ \\
$1^{1} \mathrm{~B}_{\mathrm{u}}$ & experiment & $4.41(0-0, \mathrm{v})$ & $3,5,7,10$ \\
& semiempirical & $4.46(0-0), 4.92(\mathrm{v})$ & 17 \\
& semiempirical & $4.63(\mathrm{v})$ & 28 \\
& ab initio & $4.56(0-0), 4.79(\mathrm{v})^{a}$ & $b$ \\
$2^{1} \mathrm{~A}_{\mathrm{g}}$ & experiment & $3.59(0-0)$ & 3,5 \\
& inferred expt & $3.97(\mathrm{v})$ & 29 \\
& semiempirical & $4.1(\mathrm{v})$ & 28 \\
& semiempirical & $3.44(0-0), 4.54(\mathrm{v})$ & 17 \\
& eff Hamiltonian & $3.91(0-0), 4.88(\mathrm{v})$ & 27 \\
& ab initio & $4.15(0-0), 5.21(\mathrm{v})$ & $b$ \\
$1^{2} \mathrm{~B}_{\mathrm{g}}$ & experiment & 7.8 & 30 \\
& ab initio & 7.47 & $b$
\end{tabular}

${ }^{a}$ Results in the nonaugmented basis. The vertical excitation energy in the augmented basis is $4.76 \mathrm{eV}$. ${ }^{b}$ Present results.

$2^{1} \mathrm{~A}_{\mathrm{g}}$ state, use of the same parametrization may artificially lower the covalent states.

The results for the $2^{1} \mathrm{~A}_{\mathrm{g}}$ state reveal an important point regarding qualitative differences between the ground- and excited-state potentials. Since much of the information regarding the $2^{1} \mathrm{~A}_{\mathrm{g}}$ state has been obtained based on emission experiments, it has been common to infer the vertical excitation energy from the 0-0 transition energy and the fluorescence intensity maximum. If the potentials were similar, an estimate of the vertical excitation energy could be obtained from $E_{0-0}+\left(E_{0-0}-E_{\mathrm{fl} \max }\right)$. On this basis a value of $3.97 \mathrm{eV}$ has been quoted for the vertical excitation to the $2^{1} \mathrm{~A}_{\mathrm{g}}$ state, using $E_{0-0}=3.59$ and $E_{\mathrm{f} 1 \max }=3.21 \mathrm{eV}^{29}$ However, the present results suggest that the potentials are not at all similar. The $2^{1} \mathrm{~A}_{\mathrm{g}}$ state energy changes by $1.05 \mathrm{eV}$ in proceeding from the ground-state geometry to the $2^{1} \mathrm{~A}_{\mathrm{g}}$ geometry, while the ground-state energy changes by only $0.42 \mathrm{eV}$. Thus, while our actual values (uncorrected for the error in $E_{0-0}$ ) are $E_{\text {vert }}$ $=5.21 \mathrm{eV}, E_{0-0}=4.15 \mathrm{eV}$, and $E_{\eta \max }=3.74$, the value of $E_{\text {vert }}$ which would be inferred based on our (uncorrected) calculated $E_{0-0}$ and $E_{\mathrm{flmax}}$ would be $4.56 \mathrm{eV}$ (uncorrected), in error by 0.65 $\mathrm{eV}$ relative to the actual calculated result. Similar large differences between the energy change for the $1^{1} \mathrm{~A}_{\mathrm{g}}$ and the $2^{1} \mathrm{~A}_{\mathrm{g}}$ states in going from the ground-state to the excited-state equilibrium geometries have been observed by using semiempirical or effective Hamiltonian techniques. ${ }^{17,27}$

The calculated ionization potential to the ${ }^{2} \mathrm{~B}_{\mathrm{g}}$ state is in error relative to the experimental estimate by at least $0.3 \mathrm{eV} .{ }^{30}$ The intensity maximum occurs at $7.8 \mathrm{eV}$ experimentally, but peaks at $8.0 \mathrm{eV}(>95 \% 0-0)$ and $8.1 \mathrm{eV}$ (approximately $60 \% 0-0$ ) are present which may instead represent the vertical transition. Since the errors in calculations on Rydberg states should be similar to those for cations, we expect similar accuracy in the calculated excitation energy of the Rydberg-like $2^{1} B_{u}$ state. The $2^{1} B_{u}$ state is found to be a $3 p_{x}$ Rydberg state. The calculated excitation energy to the $2^{1} B_{u}$ state ( $5.65 \mathrm{eV}$ in the augmented basis) agrees 87.

(30) Jones, T. B.; Maier, J. P. Int. J. Mass Spectrom. Ion Phys. 1979, 31, 
reasonably well with a weak feature found in the electron-energy-loss spectrum at $5.85 \mathrm{eV}^{7} \mathrm{~A}$ feature in the experimental spectrum at $6.04 \mathrm{eV}$ has been previously assigned as the $2^{1} \mathbf{B}_{u}$ state. ${ }^{7}$ Given the error in our calculated ionization potential, it is unrealistic to attempt to choose between these two features based on the calculated results.

One question which remains unanswered by the present study is the cause of the seeming disappearance of the $2^{1} \mathrm{~A}_{\mathrm{g}}$ state in the gas-phase fluorescence spectrum. Our results indicate that in relaxed excited-state geometries the $2^{1} \mathrm{~A}_{\mathrm{g}}$ state is found to lie significantly below the $1^{1} B_{u}$ state. Since the calculations are performed on the isolated molecule, the cause of this discrepancy remains unresolved. Clearly further work is required to answer this question.

\section{Conclusion}

Results are presented from ab initio CI calculations for several low-lying excited states of all-trans-octatetraene. In a vertical transition from the ground state the lowest singlet excited state is found to be of ${ }^{\prime} B_{u}$ symmetry. This state is essentially valencelike.
The second excited singlet state is a $2^{1} \mathrm{~A}_{\mathrm{g}}$ state at the ground-state geometry, the zeroth-order description of which is multiconfigurational and can be identified with the so-called "doubly excited" state found in long-chain polyenes. However, application of a correction for the $2^{1} \mathrm{~A}_{\mathrm{g}}$ state based on the estimated $0-0$ transition energy makes the $1^{1} \mathrm{~B}_{\mathrm{u}}$ and $2^{1} \mathrm{~A}_{\mathrm{g}}$ states essentially degenerate at the ground-state geometry. Relaxation of the excited-state geometries leads to the $2^{1} \mathrm{~A}$ state having the lowest $0-0$ transition energy. Reasonable agreement is found with experiment where comparisons can be made, and we predict that the $2^{1} B_{u}$ state is of $3 \mathrm{p}_{x}$ Rydberg character and lies near $5.65 \mathrm{eV}$.

Acknowledgment. We gratefully acknowledge financial support of this research by the National Insitute of Health, Grant 2 R01 GM34081-03. The calculations were performed at the Indiana University Chemistry Computational Facility, the establishment of which was in part made possible by grants from the National Science Foundation, Grants CHE-83-09446 and CHE-84-05851.

Registry No. all-trans-Octatetraene, 3725-31-3.

\title{
Fourier Transform Infrared Studies of Atomic $\mathrm{Ti}, \mathrm{V}, \mathrm{Cr}, \mathrm{Fe}, \mathrm{Co}, \mathrm{Ni}$, and $\mathrm{Cu}$ Reactions with Carbon Dioxide in Low-Temperature Matrices
}

\author{
J. Mascetti* and M. Tranquille
}

Laboratoire de Spectroscopie Molëculaire et Cristalline, UA 124 CNRS, Université de Bordeaux I, 351, Cours de la Libération, 33405 Talence Cedex, France (Received: February 11, 1987; In Final Form: October 20, 1987)

\begin{abstract}
Titanium, vanadium, chromium, iron, cobalt, nickel, and copper metal atoms were cocondensed with $\mathrm{CO}_{2}$ in neat matrices at $15 \mathrm{~K}$. Even though a large proportion of atoms do not react, new complexes with $\mathrm{CO}_{2}$ are characterized by using FTIR techniques and their infrared absorption bands are assigned. It is clearly shown that $\mathrm{CO}_{2}$ is highly bent in these complexes. The behavior of oxophile metals is different from that of other metals which spontaneously form $1: 1$ complexes with $\mathrm{CO}_{2}$. For $\mathrm{Ti}, \mathrm{V}$, and $\mathrm{Cr}$, we observe first an insertion of metallic atom into a $\mathrm{CO}$ bond, leading to the formation of the intermediate $(\mathrm{O}) \mathrm{M}(\mathrm{CO})$ molecule, followed by the $\mathrm{CO}_{2}$ fixation on this complex, to form $(\mathrm{O}) \mathrm{M}(\mathrm{CO})\left(\mathrm{CO}_{2}\right)$. The other identified products in the matrices are $\mathrm{CO}, \mathrm{M}(\mathrm{O}), \mathrm{M}(\mathrm{CO})_{n}$, and possibly a few carbonates. Most complexes are thermally unstable and decompose above $60 \mathrm{~K}$. Only $\mathrm{Fe}$ and $\mathrm{Ni}$ compounds give rise upon annealing above $80 \mathrm{~K}$ to $\mathrm{CO}_{2}$ reduction to $\mathrm{CO}$ along with the simultaneous formation of metal carbonyl complexes. Isotopic experiments with ${ }^{13} \mathrm{CO}_{2}$ and $\mathrm{C}^{18} \mathrm{O}_{2}$ allow us to conclude that the geometry of complexes is both metal and matrix dependent: in neat $\mathrm{CO}_{2}$ matrices, $\mathrm{Fe}$ and $\mathrm{Co}$ lead to a " $\mathrm{C}$ coordination", the others being fixed in a "side-on" or "end-on" manner.
\end{abstract}

\section{Introduction}

During the past 12 years there has been a considerable growth of interest in the reactions of $\mathrm{CO}_{2}$ because of the need to develop alternative hydrocarbon sources. ${ }^{1-4} \quad \mathrm{CO}_{2}$ is potentially the most abundant source of $\mathrm{C}_{1}$ chemistry but new ways must be found to activate the molecule catalytically if its potential has to be realized. Activation is one of the routes to induce inert molecules to react. Some transition-metal atoms in the low valency state form complexes with $\mathrm{CO}_{2}$. Such complexes have potential for practical application in activating $\mathrm{CO}_{2}, \mathrm{CO}_{2}$ displays several modes of coordination with metallic compounds. Bonding between $\mathrm{CO}_{2}$ and metals may lead to the following intermediate compounds: ${ }^{5}$

(1) Volpin, M. E.; Kolomnikov, I. S. Organometallic Reactions; WileyInterscience: New York, 1970; Vol. 5, p 313.

(2) Sneeden, R. P. A. Actual. Chim. 1979, 1, 31; 1979, 2, 22

(3) Lapidus, A. L.; Ping, Y. Y. Russ. Chem. Rev. 1981, 50, 63

(4) Ziessel, R. Nouv. J. Chim. 1983, 7, 613.

(5) Stankevich, I. V.; Lysyak, T. V.; Aleksandrov, G. G.; Kolomnikov, I.' S. J. Struct. Chem. 1978, 19,777.

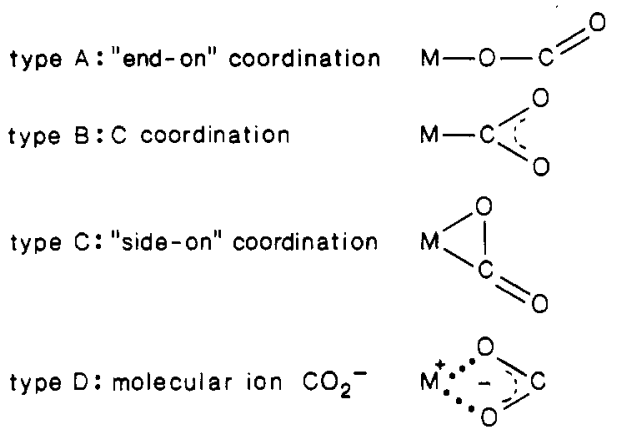

Structures of type A were proposed from ab initio calculations ${ }^{6}$ and from IR results reported by Kafafi and co-workers ${ }^{7}$ for the $\mathrm{LiCO}_{2}$ complex of $C_{s}$ symmetry. Type $\mathrm{D}$ structures were only encountered when $M$ is an alkali-metal atom and observed by RPE

(6) Sakaki, S.; Kitaura, K.; Morokuma, K. Inorg. Chem. 1982, 21, 760.

(7) Kafafi, Z. H.; Hauge, R. H.; Billups, W. E.; Margrave, J. L. J. Am. Chem. Soc. 1983, 105, 3886 . 